\title{
Solar neutrino and terrestrial antineutrino fluxes measured with Borexino at LNGS
}

\author{
Sandra Zavatarelli ${ }^{1}$ (on behalf of the Borexino Collaboration ${ }^{2}$ ) \\ I.N.F.N. Genova Italy \\ Via Dodecaneso 33, 16146 Genova (Italy) \\ E-mail: sandra.zavatarelliege.infn. it
}

Borexino is a real time liquid scintillator detector for low energy neutrino and antineutrino spectroscopy located at the Gran Sasso National Laboratories (Italy). Thanks to its unique features - extremely low background, large scintillator mass (278 tons), low energy threshold and a carefully designed muon-veto system- it is giving a substantial contribution to key issues in many physics fields, like neutrino properties, solar physics, rare decays, Earth composition and heat balance.

During the current year the Borexino collaboration efforts have been addressed to two main goals: the analysis of data set collected until December 2009 and the scintillator re-purification operations.

Results obtained in 2010 concern: the update of solar ${ }^{7} \mathrm{Be}-\boldsymbol{v}$ flux day/night asymmetry, the measurement of the solar ${ }^{8} \mathrm{~B}-v$ flux with a lower threshold down to $3 \mathrm{MeV}$, the first observation of the geoneutrino signal at $4.2 \sigma$ C.L., the measure of the European reactors antineutrino disappearence on a baseline of $1000 \mathrm{~km}$, limits on the transition probability of solar neutrinos to antineutrinos and the most stringent up-to-date experimental bounds on Pauli exclusion principle violating transitions in ${ }^{12} \mathrm{C}$ nuclei.

Purification campaigns aiming to reduce mainly the ${ }^{85} \mathrm{Kr}$ and ${ }^{210} \mathrm{Bi}$ contaminants in the scintillator are in progress. The observed reduction of background is already sizeable and it is marking the beginning of a new phase of precision solar neutrino spectroscopy with an increased sensitivity to the more elusive components of solar neutrino flux like pep and CNO neutrinos.

35th International Conference of High Energy Physics (ICHEP2010)

Paris,France

July 22-28, 2010

1 Speaker

2 Borexino Collaboration: G. Bellini, J. Benziger, S. Bonetti, M. Buizza Avanzini, B. Caccianiga, L. Cadonati, F. Calaprice, C. Carraro, A. Chavarria, F. Dalnoki-Veress, D. D’Angelo, S. Davini, H. de Kerret, A. Derbin, A. Etenko, K. Fomenko, D. Franco, C. Galbiati, S. Gazzana, C. Ghiano, M. Giammarchi, M. Goeger-Neff, A. Goretti, E. Guardincerri, S. Hardy, Al. Ianni, An. Ianni, M. Joyce, V.V. Kobychev, Y. Koshio, G. Korga, D. Kryn, M. Laubenstein, M. Leung, T. Lewke, E. Litvinovich, B. Loer, P. Lombardi, L. Ludhova, I. Machulin, S. Manecki, W. Maneschg, G. Manuzio, Q. Meindl, E. Meroni, L. Miramonti, M. Misiaszek, D. Montanari, V. Muratova, L. Oberauer, M. Obolensky, F. Ortica, M. Pallavicini, L. Papp, L. Perasso, S. Perasso, A. Pocar, R.S. Raghavan, G. Ranucci, A. Razeto, A. Re, P. Risso, A. Romani, D. Rountree, A. Sabelnikov, R. Saldanha, C. Salvo, S. Schonert, H. Simgen, M. Skorokhvatov, O. Smirnov, A. Sotnikov, S. Sukhotin, Y. Suvorov, R. Tartaglia, G. Testera, D. Vignaud, R.B. Vogelaar, F. von Feilitzsch, J. Winter, M. Wojcik, A. Wright, M. Wurm, J. Xu, O. Zaimidoroga, S.Zavatarelli and G. Zuzel 


\section{Introduction}

Borexino has opened a new chapter in the experimental history of solar neutrinos making feasible the solar $v$ 's spectroscopy in real time down to $200 \mathrm{keV}$. This was possible employing a liquid scintillator technique that offers several advantages: the light yield is a factor 50 higher than the Cherenkov one and the very low solubility to ions and metal impurities makes it possible to reach unprecented levels of radiopurity.

The detector is located at the Gran Sasso National Laboratories (LNGS) at a depth of 3800 m.w.e: its active mass consists of 278 tons of pseudocumene (PC) doped with $1.5 \mathrm{~g} / 1$ of PPO [1]. The scintillation light is detected via 22128 " photomultipliers tubes. Additional 208 " PMT's detect the Cherenkov light radiated by muons in the water shield, serving as muon veto.

The neutrino signal is detected through the elastic scattering on electrons in the scintillator. Electron antineutrinos are conversely detected through the inverse beta decay reaction $v_{\mathrm{e}}+\mathrm{p} \rightarrow$ $\mathrm{e}^{+}+\mathrm{n}$. The cross section for this process is much higher than for $v$-e elastic scattering and the coincidence between the prompt signal of positron annihilation and the delayed signal due to the neutron capture on a proton $(\tau \sim 256 \mu \mathrm{s})$ gives a clean event signature and it allows for the detection of relatively few events with high significance.

In the present work all the recent results based on a 2.5 years data statistics (May 2007Dec 2009) will be reviewed and future science goals discussed.

\section{The measure of solar neutrino fluxes}

The Sun is an intense source of electron neutrinos, produced in nuclear reactions of the $p-p$ chain and of the CNO cycle. They provide a unique probe for studying both nuclear fusion reactions that power the Sun and the fundamental properties of neutrinos. Solar neutrinos have been studied for 30 years by means of radiochemical and water Cherenkov detectors and brought to the discovery of the $v$ flavour oscillations.

\subsection{The ${ }^{7}$ Be- $v$ signal: flux and day/night asymmetry}

The primary goal of Borexino is the real-time measurement of the monoenergetic ( 862 $\mathrm{keV}$ ) neutrino flux originating from the electrons capture on ${ }^{7} \mathrm{Be}$ in the Sun's core.

A $10 \%$ flux measurement has been published in 2008 based on a 192 days statistics [2]: the ${ }^{7} \mathrm{Be}-\mathrm{v}$ interaction rate is $(49 \pm 3 \pm 4) \mathrm{ev} /$ (day 100t) and it corresponds to a $v_{\mathrm{e}}$ survival probability $\mathrm{P}_{\mathrm{ee}}$ of $(0.56 \pm 0.10)$. Therefore this result on one hand confirms the MSW-LMA $v$ oscillation scenario and on the other hand it provides the first direct $\mathrm{P}_{\mathrm{ee}}$ measurement in the low energy vacuum regime.

After that, a huge experimental and analysis effort has been done in order to decrease the errors associated to the measurement of the ${ }^{7} \mathrm{Be}$ signal rate. Careful calibration campaigns were performed from October 2008 to July 2009 to decrease the fiducial volume and the detector energy response uncertainties and they provided the elements to tune the Monte Carlo code 
describing the detector: a very precise $\mathrm{MC}$ based fitting procedure has been developed and a new result with a $5 \%$ precision it is going to be published based on a 3 year statistics.

A renewed analysis of the day and night spectra showed no significant day-night asymmetry in the ${ }^{7} \mathrm{Be}$ flux. Data corresponding to a total lifetime of 789 days have been analyzed. The day-night asymmetry is defined as $A_{d n}=2(N-D) /(N+D)$ where $D$ and $N$ are the neutrino fluxes during day and night time. We get $A_{d n}=(0.007 \pm 0.073)$ (stat), consistent with zero.

\subsection{The ${ }^{8} \mathrm{~B}-v$ fluxes and the survival probability in the vacuum-matter oscillation transition}

The extreme radiopurity of Borexino combined with the efficient software rejection of cosmogenic background allows the study of the recoiled electron spectrum induced by ${ }^{8} \mathrm{~B}$ solar $v$ down to the energy threshold of $3 \mathrm{MeV}$ [5]. This value is mainly due to the presence at lower energies of a large background coming from penetrating $\gamma$ rays emitted by ${ }^{208} \mathrm{Tl}$ decay in the PMT's material.

The energy spectrum of events surviving all cuts and after the ${ }^{208} \mathrm{Tl}$ and ${ }^{11} \mathrm{Be}$ statistical subtraction is shown in Fig.1-left. The measured overall flux (above $3 \mathrm{MeV}$ ) is $\Phi_{8 B}^{E S}=(2.4 \pm 0.4 \pm 0.1) \cdot 10^{6} \mathrm{~cm}^{-2} \mathrm{~s}^{-1}$ [3] and the equivalent $v_{\mathrm{e}}$ survival probability, is $(0.29 \pm 0.10)$ at the effective energy of $8.9 \mathrm{MeV}$.
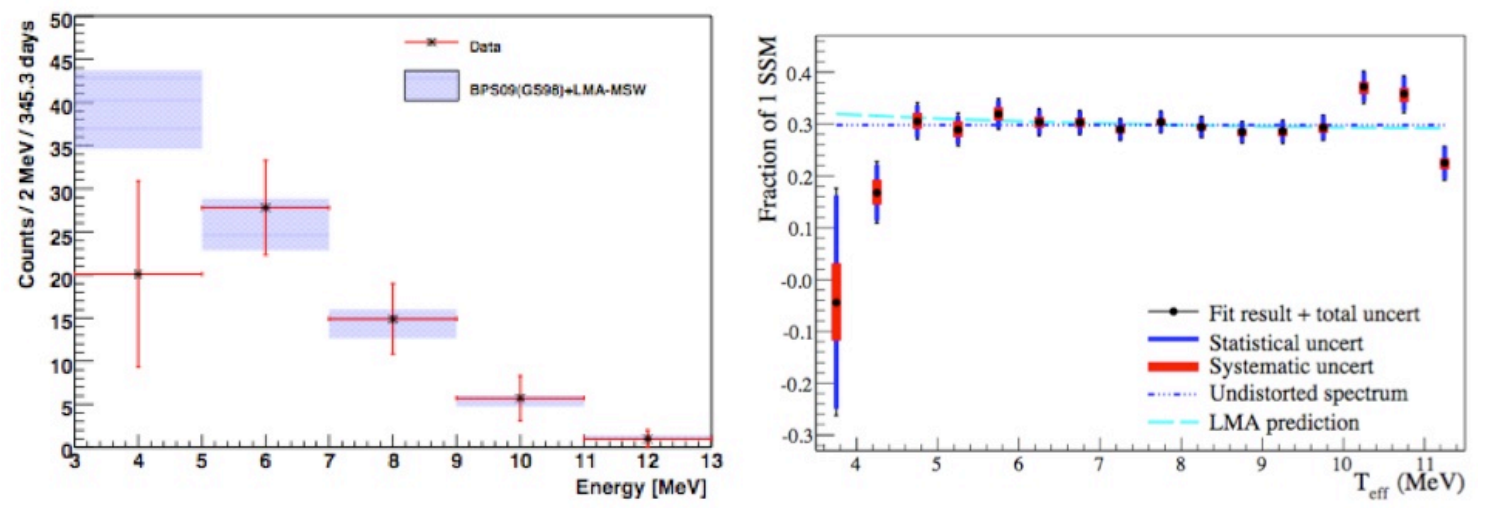

Fig. 1 Left: ${ }^{8} \mathrm{~B}-v$ spectrum measured by Borexino, compared to the expectations based on SSM BP09 (GS98) in MSW-LMA scenario. Right: ${ }^{8} \mathrm{~B}-\boldsymbol{v}$ energy spectrum measured by SNO [4]

Strong efforts are presently performed in order to decrease the energy threshold of the analysis and/or to increase the fiducial volume. The small event deficit in the low energy bin of ${ }^{8} \mathrm{~B}$ flux is in agreement with the result from SNO [4] (Fig.1-right) and, if confirmed, it could be the hint of a new physics beyond the LMA-MSW solution to the solar neutrino problem.

\section{Antineutrinos from the Earth, from European reactors and unknown sources}

Geo-neutrinos are electron antineutrinos produced in $\beta$-decays of ${ }^{40} \mathrm{~K}$ and of several nuclides in the ${ }^{238} \mathrm{U}$ and ${ }^{232} \mathrm{Th}$ chains that are naturally present in the Earth and that can be used as a unique probe of the Earth interior. By measuring their flux and spectrum, it is possible to reveal the distribution of long-lived radioactivity in the Earth and to assess the radiogenic contribution to the total heat balance of our planet. These pieces of information, in turn, are 
critical for the understanding of complex processes such as the generation of the Earth's magnetic field, mantle convection, and plate tectonics.

With a 252.6 ton-yr fiducial exposure after all selection cuts $9.9_{-3.4}^{+4.1}$ geo-neutrino events were observed which correspond to a geo-neutrino flux of $7.2_{-2.4}^{+2.9} \mathrm{TNU}$ [6]. From the $\ln \mathcal{L}$ profile the statistical significance of the geo-neutrino observation is $99.997 \%$ C.L. (4.2 $\sigma$ ). The observed prompt energy spectrum above $2.6 \mathrm{MeV}$ is compatible with that expected from European nuclear reactors and it allows tests for the first time of antineutrino propagation properties on a baseline of about $1000 \mathrm{~km}$. Our measurement excludes the non-oscillation hypothesis at $99.60 \%$ C.L. and it rejects the hypothesis for an active geo-reactor in the Earth core with a power above $3 \mathrm{TW}$ at $95 \%$ C.L. [5].

By studying the antineutrino signal a limit of $1.310^{-4}$ (90\% C.L.) for $E_{\bar{v}}>1.8 \mathrm{MeV}$ on the transition probability of solar $v$ to anti- $v$ has been obtained together with the best differential limits on antineutrino fluxes from unknown sources for energies below $17.8 \mathrm{MeV}$ [6].

\section{PEP violating transition on ${ }^{12} \mathrm{C}$}

The Pauli exclusion principle (PEP) has been tested searching for $\gamma, n, p$ and $\beta$ emitted in non-Paulian transition of ${ }^{1} \mathrm{P}_{3 / 2}$-shell nucleons to the already filled ${ }^{1} \mathrm{~S}_{1 / 2}$ shell in ${ }^{12} \mathrm{C}$ nuclei. The following most stringent up-to-date experimental bounds have been established [7]: $\tau\left({ }^{12} \mathrm{C} \rightarrow\right.$ $\left.{ }^{12} \tilde{C}+\gamma\right) \geq 5 \cdot 10^{31} y r, \tau\left({ }^{12} C \rightarrow{ }^{11} \tilde{B}+p\right) \geq 8.9 \cdot 10^{29} y r, \tau\left({ }^{12} C \rightarrow{ }^{11} \tilde{C}+n\right) \geq 3.4 \cdot 10^{30} y r, \tau\left({ }^{12} C \rightarrow{ }^{12} \tilde{N}+e^{-}\right.$ $\left.+\tilde{v}_{e}\right) \geq 3.1 \cdot 10^{30} y r, \tau\left({ }^{12} C \rightarrow{ }^{12} \tilde{B}+e^{+}+v_{e}\right) \geq 2.1 \cdot 10^{30} y r$ all at $90 \%$ C.L..

\section{Conclusions}

Borexino has completed its first scientific phase (May2007-May2010) providing a first real time measurement of the ${ }^{7} \mathrm{Be}-v$ flux and day-night asymmetry and the lower threshold ${ }^{8} \mathrm{~B}-v$ rate. The signal due to Earth antineutrinos has been clearly detected too. Scintillator purifications are running since June 2010 and the already apparent reduction of backgrounds is marking the beginning of a new phase of precision solar neutrino spectroscopy for Borexino.

\section{References}

[1] G. Alimonti et al. (Borexino Collaboration), Nucl. Instrum. Meth. A 600 (2009) 568

[2] C. Arpesella et al. (Borexino Collaboration), Phys. Rev. Lett. 101 (2008) 091302

[3] G. Bellini et al. (Borexino Collaboration) Phys. Rev. D 82 (2010) 033006

[4] B. Aharmim (SNO Collaboration) Phys. Rev. C 81 (2010) 055504

[5] G. Bellini et al. (Borexino Collaboration), Phys. Lett. B 687 (2010) 299

[6] G. Bellini et al. (Borexino Collaboration),http://arxiv.org/abs/1010.0029 submitted to Phys. Lett. B

[7] G. Bellini et al. (Borexino Collaboration), Phys. Rev. C 81 (2010) 034317 